\title{
The bidirectional improvement of level set method topology optimization
}

\author{
Dongyue Qu ${ }^{1}$, a, Fanbing $\mathrm{Liu}^{1, \mathrm{~b}}$, Chunhua Zhao ${ }^{1}$ and Xiuming $\mathrm{Li}^{1}$ \\ ${ }^{1} S$ chool of Harbin, engineering University, Harbin 150000, China \\ aqudongyue@hrbeu.edu.cn, bliufanbing@hrbeu.edu.cn
}

Keywords: reaction diffusion equation, bidirectional optimization, level set function.

Abstract. This paper research the Topology optimization based on level set method. Existing level set topology optimization method based on the reaction diffusion equation does not satisfy the volume constraints and convergence of objective function when solve some model optimization. According to this phenomenon, this paper proposes a new level set topology optimization algorithm based on the reaction diffusion equation, this algorithm can control the direction of the level set function and do bidirectional optimization which the objective function under the condition of volume constraint can reduce material or increase the material. Eventually, the results of optimization can become steady.

\section{Introduction}

At present, continuous structure topology optimization has become an important research field of engineering application. Level set method was proposed by Osher and Sethian [1] and used to study the dynamic interface tracking as a numerical method. As shown in Fig. 1, the main idea of this method is to use zero level surface (lines) of high dimension level set function to express the represent evolution of surface(curve) implicitly, and curve or surface boundary motion analysis and tracking will be obtained by solving the level set equation.Using sensitivity analysis of the level set function to solve the structure optimization which has been proposed by Allaire [2].The level set function is firstly introduced into the field of structural topology optimization by Sethian [3]. An optimization model was proposed by Wang [4], which established the objective function by the most minimized structure compliance and build the volume limit as the constraint condition. Challis [5] using a discrete level-set method to solve structural topology optimization and present a matlab code. The reaction diffusion equations was introduced to solve the level set function method for structural topology optimization by Masaki Otomori [6] and create a new topology optimization method, this article is based on the method to carry out relevant bidirectional improve optimization.

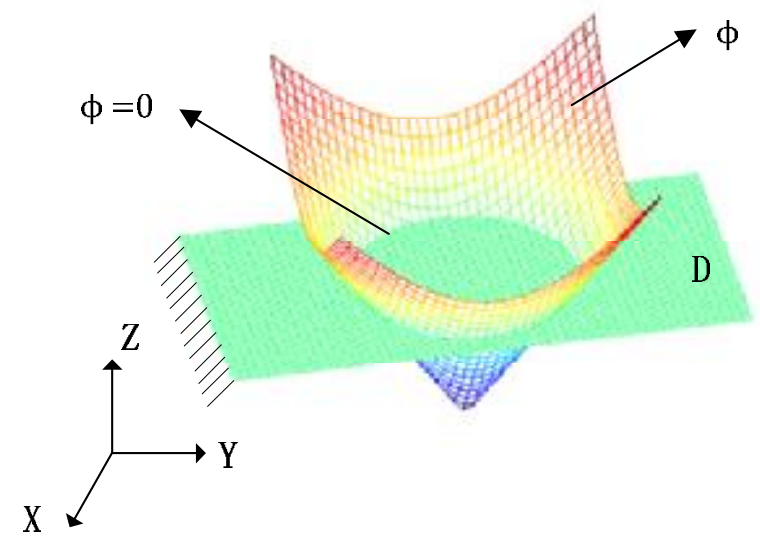

Fig.1 The level set function diagram

As shown in Fig. 1, in the level set topology optimization, we define $\phi$ as the level set function, D as design domain. The positive value of $\phi$ represents the material area and the negative value represents the empty domain .Besides zero represents the boundary of structure. 


$$
\left\{\begin{array}{cc}
\phi(x)>0 & \text { if } x \in \Omega \\
\phi(x)=0 & \text { if } x \in \partial \Omega . \\
\phi(x)<0 & \text { if } x \in D / \Omega
\end{array}\right.
$$

\section{Topology optimization formula}

As shown in Fig. 2, D and $\Omega$ respectively represent the mixed design area and the material design area, the objective function of the design is to minimize structural compliance, and get a reasonable mechanical structure. When the force of $\Gamma_{\mathrm{i}}$ is applied at the boundary $\Gamma_{\mathrm{f}}$, the displacement is zero at the boundary $\Gamma_{u}$.

$$
\begin{aligned}
& \min _{\phi} \quad J=\int_{\Gamma} F_{i} u_{i} d \Gamma \\
& \text { st. } G=\int_{\Omega} d \Omega-V_{\max } \leq 0 \\
& \operatorname{div}\left(E_{i j k l} u_{k, l}\right)=\operatorname{div}\left(\sigma_{i j}\right)=0 \quad \text { on } \Omega . \\
& u_{i}=0 \quad \text { in } \Gamma_{u} \\
& F_{i}=F_{i}^{-} \quad \text { in } \Gamma_{f}
\end{aligned}
$$

Where $V_{\max }$ is volume constraint upper limit, $E_{i j k l}$ is the elastic tensor, $u_{i}$ is displacement and equal to $0, F_{i}$ is the external force acting at the boundary $\Gamma_{f} \operatorname{div}\left(E_{i j k l} u_{k, l}\right)=\operatorname{div}\left(\sigma_{i j}\right)=0$ represent that there is no other external load on the material area. Using Lagrange multiplier algorithm transform above formulas into a unconstrained Lagrange formula is as follows:

$$
\bar{J}=\int_{\Gamma} F_{i} u_{i} d \Gamma+\int_{\Omega} \tilde{u_{i}} \operatorname{div}\left(E_{i j k l} u_{k, l}\right) d \Omega+\lambda\left(\int_{\Omega} d \Omega-V_{\max }\right) .
$$

Where $J^{-}$as the new objective function and $\tilde{u}_{i}$ as the Lagrange multipliers.

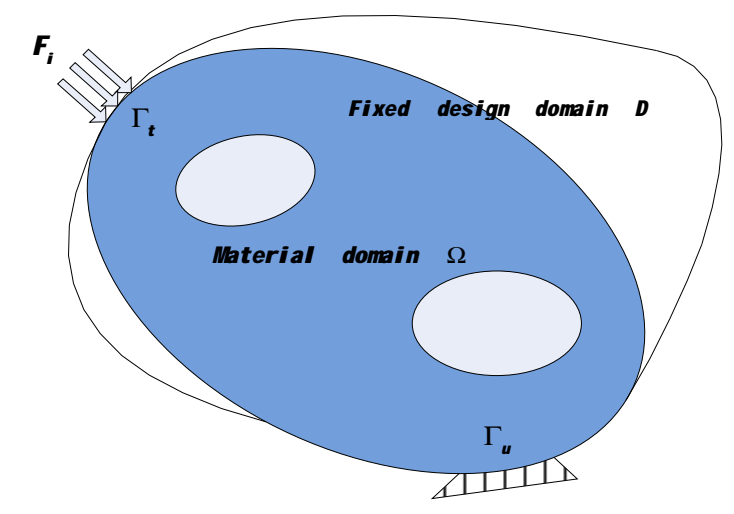

Fig.2 design domain and boundary conditions diagram 


\section{Reaction diffusion equation}

The reaction diffusion equation is used to solve the level set function , as shown below:

$$
\left\{\begin{array}{lc}
\frac{\partial \phi}{\partial t}=C d_{t} J^{-}+\tau \nabla^{2} \phi & \text { in } D \\
\phi=0 & \text { on } \partial D
\end{array} .\right.
$$

Where, the purpose of parameter $\mathrm{C}$ is to normalize the sensitivities of level set function, the diffusive term $\nabla^{2} \phi$ is used to insures the optimization has enough smoothness, the $\tau$ is a regularization parameter, its value could impact the degree of the diffusive, where $\mathrm{C}$ is defined as the follow:

$$
C=\frac{\int_{D} d \Omega}{\int_{D} d_{t} F \mid d \Omega} .
$$

Here, Masaki Otomori [6] provide a detailed derivation process about above formulas.

\section{Bi-direction optimization improvement}

We have a bi-direction optimization improvement based on the level set method based on a reaction diffusion equation, the reason of it is that the relevant theoretical method in the previous paper [6] is merely content to the volume unidirectional decrease during the program running. However, there are two necessary convergence conditions, one is volume constraint, and another is the relative stability of the objective function value. During the program running, there is a kind of situation is that when the volume constraint satisfied, but the stability of objective function value isn't met, the iteration will go continuous in the direction of reducing volume. From that, the volume constraint can't be satisfied, eventually leading to failure. Aiming at this phenomenon, we improved the relative theory and Program. When the value of the current volume is less than the value of the volume set, the program will change the direction of the corresponding optimization. By this way, it can make the program run towards to the direction of the volume increased. Eventually, the results can satisfy the stability of objective function value and volume constraints. $\lambda$ is the Lagrange multiplier of the augmented Lagrangian equation, it will be updated by adding the parameter M,as shown in the following formula.

$$
\lambda=M \frac{\int_{D} d_{t} J^{-} d \Omega}{\int_{D} d \Omega} \exp \left[p\left(\frac{G}{G_{\max }}+d\right)\right]
$$

There the parameters $\mathrm{p}$ and $\mathrm{d}$ regulate the position of the curve, and the flow diagram of the bidirectional improvement as the Fig.3. 


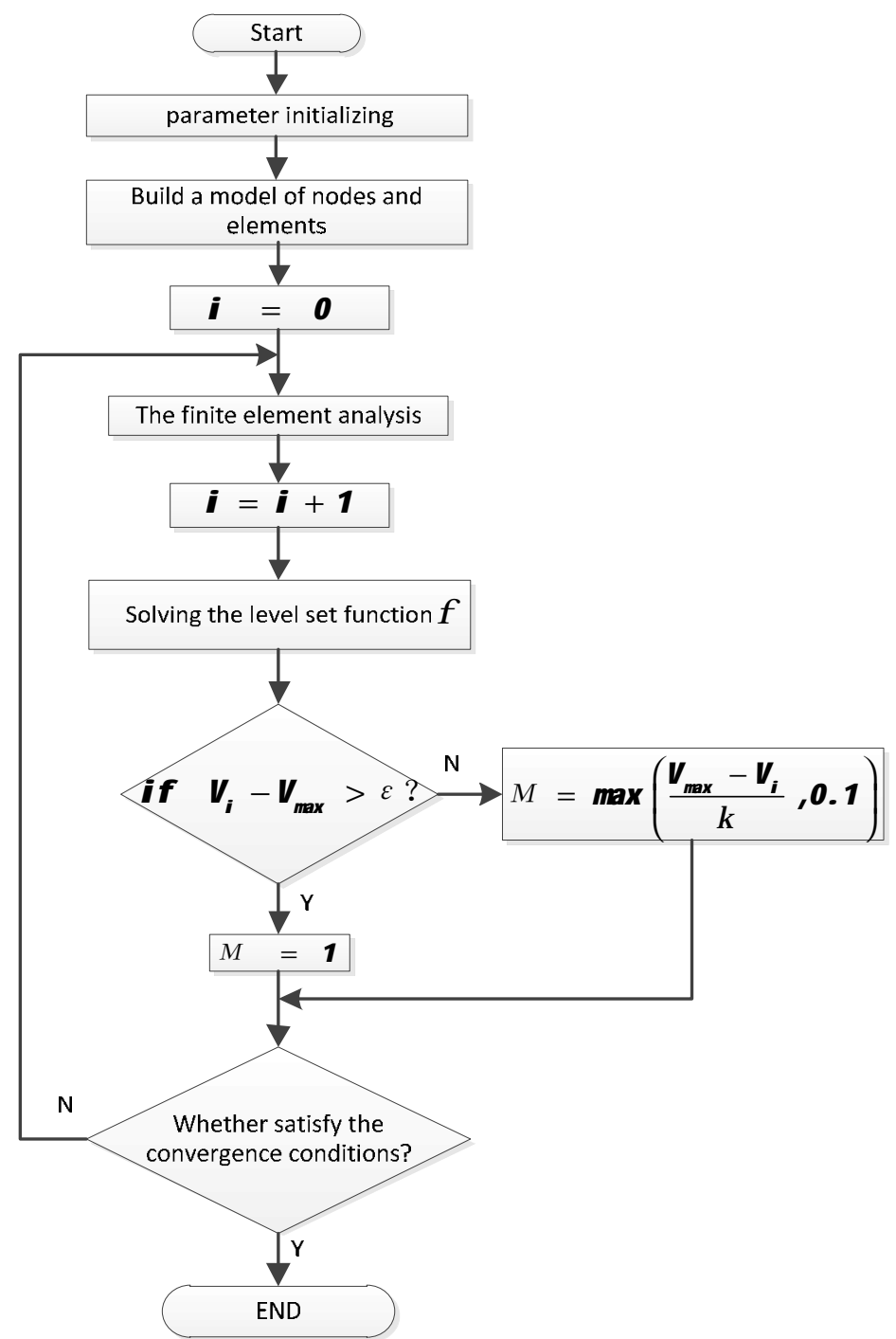

Fig.3 Flow diagram of the bidirectional improvement

Where, we define $i$ as the iteration steps, $V_{i}$ as the ratio of residual volume after it $h$ iteration, and $\mathrm{V}_{\max }$ as the setted value of volume constraint. Besides, the value of $\mathrm{K}$ is $0.3, \varepsilon$ is equal to 0.1 , while $V_{i}-V_{\max }>\varepsilon, M$ is equal to 1 , Otherwise, $M$ is equal to $\max \left(\frac{V_{\max }-V_{i}}{k}, 0.1\right)$.

\section{Numerical examples}

Case 1: Cantilever beam

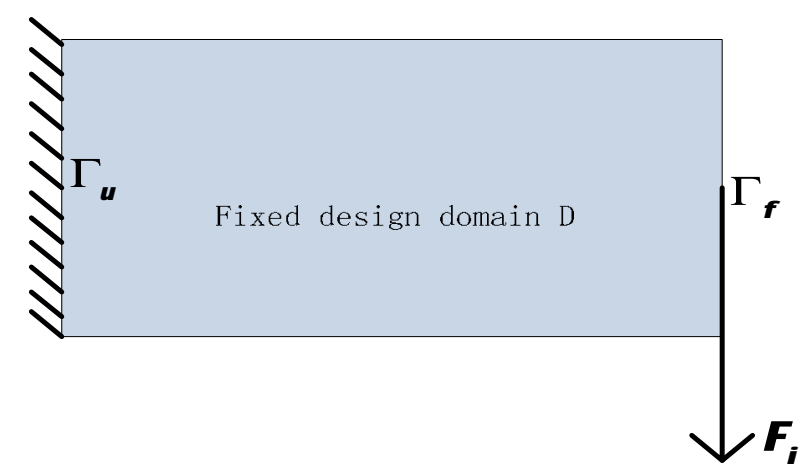

Fig.4 Structure diagram of the cantilever beam 
Fig.4 shows the displacement constraints and loads of cantilever beam. The degree of discretization is submitted to the following mesh parameters: 60x30. The upper fraction of the volume $V_{\max }$ is equal to 0.5 . We have a comparison of the results between the unimproved and bidirectional improvement as follows Fig.5, and the final result as Fig.6.
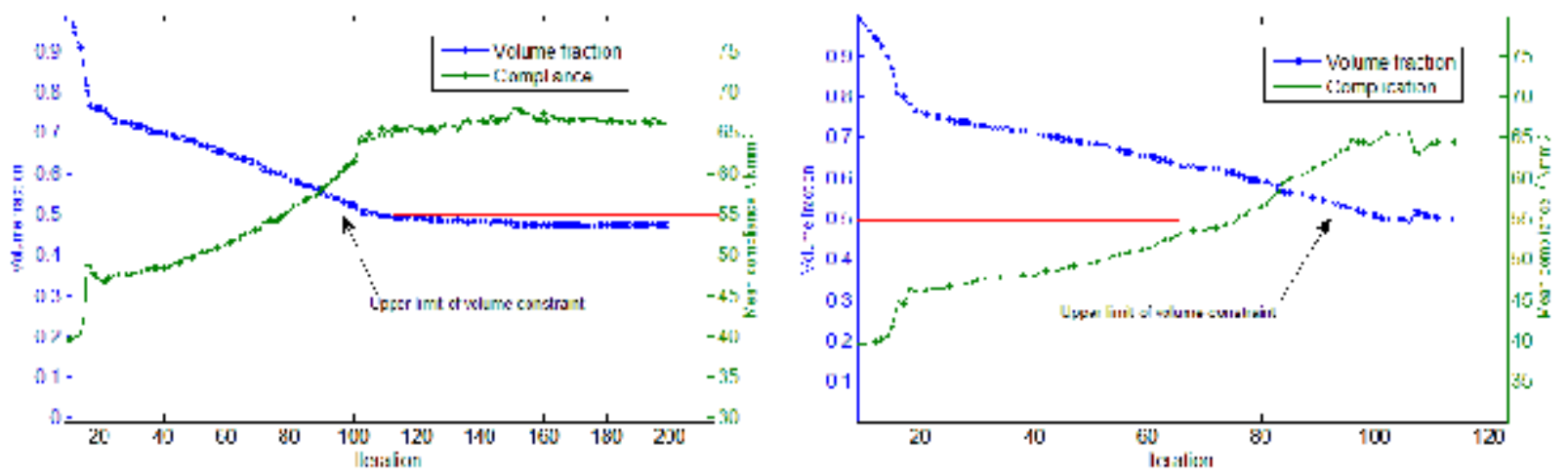

Fig.5 Evolution histories before improvement (left) and with Bidirectional improvement (right)

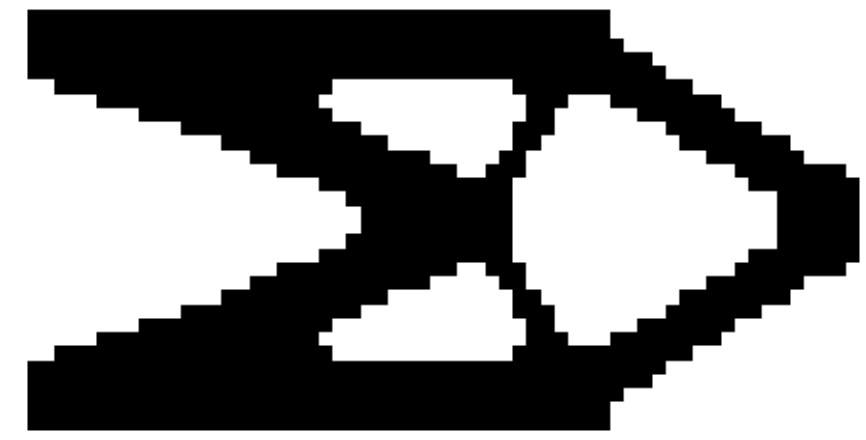

Fig.6 The topology structure with Bidirectional improvement

\section{Case 2: Michell beam}

Fig.7 shows the displacement constraints and the loads of the Michell beam. Besides, the degree of discretization is submitted to the following mesh parameters: 100x50. The volume fraction $V_{\max }$ is equal to 0.5 , The load $\mathrm{F}$ is equal to 5 . We have a comparison of results between the without the improvement and bidirectional improvement about level set method as follows Fig.8, and the final result as Fig.9.

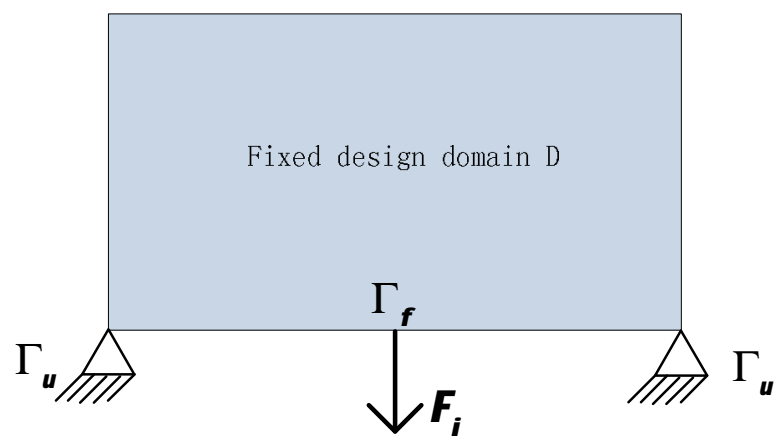

Fig.7 Structure diagram of the Michell beam 

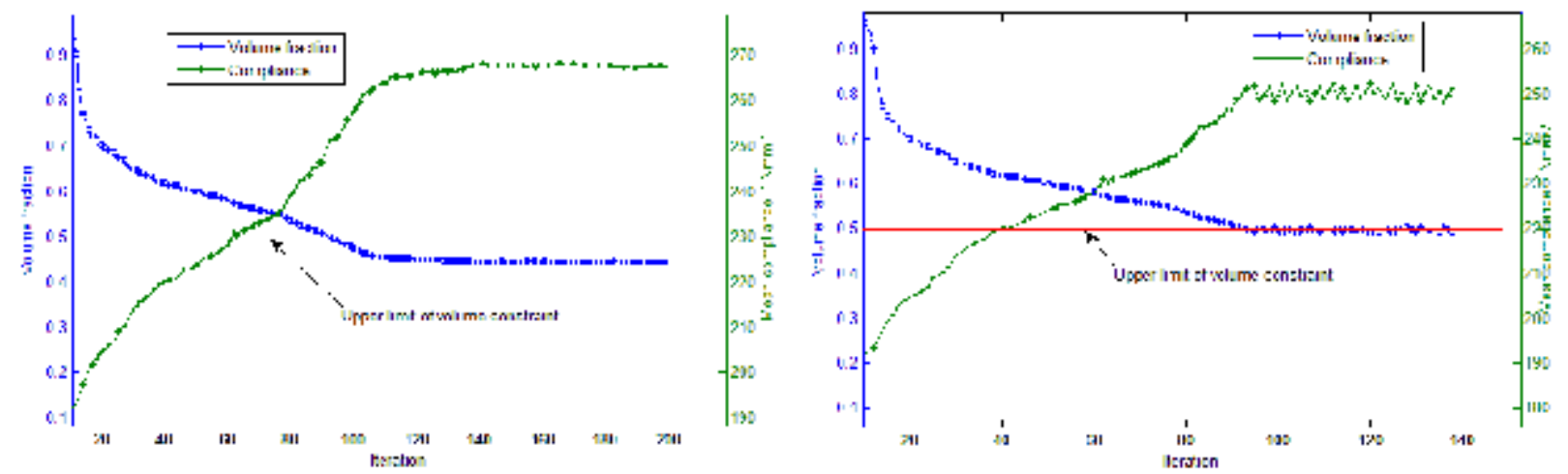

Fig.8 Evolution histories before improvement (left) and with Bidirectional improvement (right)

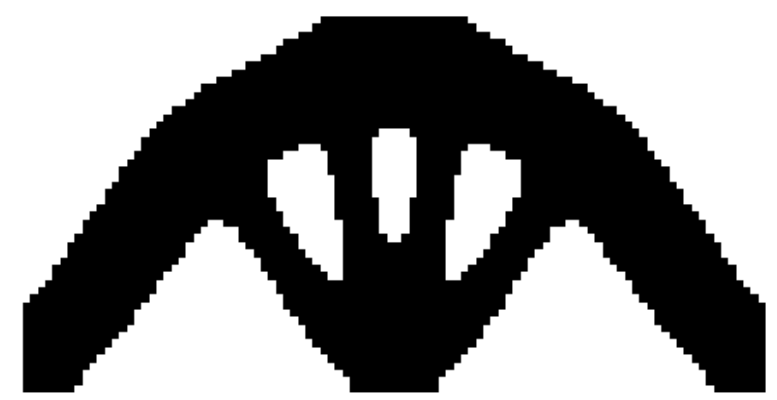

Fig.9 The topology structure with Bidirectional improvement

In the Fig.5 and Fig.8, the red line represent the value of volume constraint, and we can see the value of volume fraction is equal to the value of volume constraint with Bidirectional improvement.

\section{Conclusions}

we can get the solution from above examples, it is very difficult to satisfy the volume constraint without the improvement in the optimization, the results of the volume is less than the volume constraint value ultimately, in this paper, we by improving the original optimization program and using bidirectional improvement of topology optimization method, it enables the topology optimization to ensure volume constraint, at the same time, also achieve stability of the complaisant finally.

\section{References}

[1] S. Osher and J.A. Sethian: Fronts propagating with curvature dependent speed: algorithms based on Hamilton-Jacobi formulations. Journal of Computational Physics. Vol. 79(1988), p.12-49.

[2] G. Allaire, F. Jouve and A.M. Toader: Structural optimization using sensitivity analysis and a level-set method. Journal of Computational Physics. Vol. 194(2004), p.363-393.

[3] J.A. Sethian and A. Wiegmann: Structural boundary design via level set and immersed interface methods .Journal of Computational Physics. Vol.163(2000), p. 489-528.

[4] M.Y. Wang, X.M. Wang and D.M. Guo: A level set method for structural topology optimization. Computer Methods in Applied Mechanics and Engineering, Vol.192(2003), p.227—246.

[5] V.J. Challis: A discrete level-set topology optimization code written in MATLAB. Structural and Multidisciplinary Optimization. Vol.41(2010), p.453-464.

[6] M. Otomori, T. Yamada, K. Izui and S. Nishiwaki: Matlab code for a level set-based topology optimization method using a reaction diffusion equation. Structural and Multidisciplinary Optimization. Vol.51(2015), p. 1159-1172. 